\title{
The experiences of mothers and caregivers on feeding practices of children under the age of five years with undernutrition in Oshikoto region, Namibia
}

\author{
Ester Mulenga, Hans Amukugo, Anna Shilunga \\ Faculty of Health Science, School of Nursing, University of Namibia, Oshakati campus, Namibia
}

Received: February 23, 2018

DOI: $10.5430 /$ ijh.v4n2p20

\author{
Accepted: May 23, 2018 \\ Online Published: May 30, 2018
}

\begin{abstract}
Background: Undernutrition in children under the age of five years is prevalent in the developing countries which increase the risk of morbidity and mortality among such age group. Lack of information related to nutrition and feeding practices as well as other factors such as employment and pressure from family and community members cause mothers and caregivers of children under the age of five years to use inappropriate methods of feeding which contribute to undernutrition.

Purpose: The aim of this article is to relate the experiences of mothers and caregivers on feeding practices of children under the age of five years in Oshikoto region, Namibia.

Methods: A qualitative study design was utilized to explore and describe the experiences of mothers and caregivers on feeding practices of children under the age of five years. The study population comprised of mothers and caregivers of children diagnosed with undernutrition either severe or moderate, admitted in pediatric units or registered on Nutritional Assessment Counseling and Support programme. A purposive sampling was used to select participants and a total number of fifteen mothers/caregivers were interviewed. The data were transcribed verbatim and analysed using Tesch steps.

Results: The study revealed that mothers and caregivers experienced lack of information related to nutrition and feeding practices and other contextual constraints which resulted in the utilization of suboptimal feeding practices of children under the age of five years.

Conclusions: Due to challenges experienced by mothers and caregivers, they need to be empowered with knowledge on nutrition and feeding practices of children under the age of five years.
\end{abstract}

Key Words: Caregivers, Experiences, Feeding practices, Mothers, Undernutrition

\section{INTRODUCTION}

Feeding practices of children under the age of five years plays a big role in their growth and development. Although the Ministry of Health and Social services in Namibia has developed a policy on infant and young child feeding, poor feeding practices still remain a problem as only $49 \%$ of chil- dren less than six months were exclusively breastfed and $28 \%$ were breastfed till two years. ${ }^{[1]}$ About $26 \%$ of infant were bottle-fed and this type of feeding start as early as two to three months, some children are given complementary feeding early. Thirteen percent (13\%) and 16\% of children less than six months started eating other food and consumed

${ }^{*}$ Correspondence: Ester Mulenga; Email: emulenga@unam.na; Address: Faculty of Health Science, School of Nursing, University of Namibia, Oshakati campus, Namibia. 
water respectively. ${ }^{[1]}$ In some cases, the food given to these children are not safe as they are not prepared hygienically and do not have adequate nutrients since they rarely received fruit, vegetables, meat, fish and chicken. ${ }^{[1]}$

Researchers in African countries found out that insufficient knowledge of mothers and caregivers was one of the causes of poor feeding practices. ${ }^{[2]}$ In most cases, mothers and caregivers lack knowledge related to breastfeeding duration, when to start complementary feeding and appropriate food for children under the age of five years. ${ }^{[2]}$ Poor feeding practices contribute to the development of undernutrition especially in children under the age of five years. About $24 \%$ of children under the age of five years are stunted, $6 \%$ wasted and $13 \%$ are underweight in Namibia. ${ }^{[1]}$ Oshikoto region is among top five regions with children under five years affected by undernutrition as $26 \%$ are stunted, $9 \%$ wasted and $21 \%$ are underweight.

Although people in that region produce their own food, what they experienced with regard to feeding practices of their children was not known, so there was a need to explore the experiences of mothers and caregivers on feeding practices of children under the age of five years who developed undernutrition.

Due to lack of information, employment and pressure from family members, mothers stop breastfeeding early and they introduced complementary feeding before six months. Mothers and caregivers also feed their children less in frequency, since some children are being fed three times a day while they have already stopped breastfeeding. They also forced their children to eat when if the child is refusing to eat.

This article will be structured as follow, first lack of information related to nutrition and feeding practices as well as suboptimal feeding will be reviewed. Subsequently the article will report on the methodology, results and discussions of the study and it will conclude with recommendations that need to be implemented to resolve the issue of inadequate information.

\section{LITERATURE REVIEW}

\subsection{Lack of information related to nutritional and feed- ing practices}

Good nutrition is required for the children to grow and develop. Mothers and caregivers need to have knowledge on feeding practices and nutrition to be able to feed their children properly. A study conducted in China reported that the diet given to children lacked food rich in micronutrients as mothers and caregivers do not understand the importance of nutrition. ${ }^{[3]}$

Published by Sciedu Press
It was indicated by other researchers that children under the age of five years are not fed with adequate variety of food as caregivers lack information related to nutrition. ${ }^{[4]}$ Mothers and caregivers lack information related to nutrition, because they were not provided with such information by the health workers. ${ }^{[5]}$ As a result, mothers/caregivers felt that whatever they feed their children contains sufficient nutrients. Some researchers reported that shortage of knowledge on kinds of food suitable for children under the age of five years contributed to the mothers and caregivers to choose unsuitable food for the young children. ${ }^{[6]}$

Shortage of information with regard to feeding practices and pressure from relatives are some of the obstacles which prevent mothers and caretakers to feed their children correctly and leads to children to develop undernutrition. ${ }^{[7]}$ They furthermore indicated that mothers and caregivers have inadequate knowledge on breastfeeding frequency, when to stop breastfeeding and when to start complementary feeding. ${ }^{[7]}$ Other researchers reported that mothers lack knowledge and understanding on the benefits of breastfeeding resulted in lack of exclusive breastfeeding. ${ }^{[8,9]}$

\subsection{Suboptimal feeding practices}

Lack of knowledge and other factors caused mothers and caregivers to utilise suboptimal feeding practices. Suboptimal feeding practices are those feeding practices which do not comply with the standards set by World Health Organisation (WHO).

WHO set standards for optimal feeding practices which include exclusive breastfeeding for six months, then adding complementary feeds and continuing breastfeeding till two years or beyond. ${ }^{[10]} \mathrm{WHO}$ also developed guidelines to be followed when starting complementary feeding which include commencing with small quantity of food at six months, then increase slowly the food consistency and variety. ${ }^{[1]} \mathrm{Var}-$ ious researchers reported that some children were not exclusively breastfed since they were offered complementary feeding early. ${ }^{[12-14]}$ These researchers furthermore stated that some children were offered complementary food at the age of 3-4 months. Mothers and caregivers commence complementary feeding as they think that breast milk is not enough to assist their children to grow. ${ }^{[15]}$ Some food given to children are poor in nutrients especially protein and micronutrients. ${ }^{[6]}$ Lack of exclusive breastfeeding and poor complementary feeding put the child at risk of irreversible stunting, poor cognitive development and infectious conditions such diarrhea and acute respiratory infections. ${ }^{[16,17]}$

Mothers sometimes stop breastfeeding early. Such early stopping of breastfeeding has a negative effect even on im- 
mune system of the child since breastfeeding contain the immune-protective factors. ${ }^{[13]}$ Some researcher reported that some mothers cease to breastfed before six months or within twelve months after birth. ${ }^{[8,18,19]}$ This resulted in children to develop undernutrition even before their first birthday. When children commence complementary feed, they need to be fed responsively, but not to be forced to eat. Responsive feeding is defined as giving food to the child slowly and tolerantly while encouraging such child to eat. ${ }^{[20]}$ However, some caregivers attempt to control the feeding of their children by forcing them to eat. ${ }^{[21]}$ On the other hand some caregivers use opposite method whereby slight effort is done to motivate and encourage the children to eat. ${ }^{[21]}$

One of the optimal feeding practices standards set by WHO is that as the child grows old, feeding needs to be increased gradually in frequency and consistency to prevent decreased resistance to infections. ${ }^{[22]}$

According to WHO, a child who is not breastfeeding needs to eat at least five meals or more per day. However, some mothers feed their children less than three times per day as some children were fed according to family meal schedule. ${ }^{[6,23]}$ Feeding the children less in frequency cannot meet the nutritional needs of the child who is still in the process of growing and developing.

Dietary diversity plays a vital role in the provision of adequate nutrients to the child. Dietary diversity refers to the amount of food groups eaten per day. ${ }^{[24]}$ A study conducted in Nepal found that only one child out of three was given food from different groups. ${ }^{[25]}$ Another study done in South Ethiopia indicated that children under the age of five years were given dietary diversity less than what WHO has suggested. ${ }^{[26]}$ These children were offered food made from grains and they were not fed with fruits and vegetables which deprive them from getting vitamins and minerals. ${ }^{[26]}$ Some mothers and caregivers believe that some vegetables are not good for children as they are difficult to digest and can cause diarrhea. ${ }^{[24]}$ Inadequate provision of macro and micronutrients cause stunted growth in children and it affects their intellectual capacities. ${ }^{[27]}$

\subsection{Objective}

The objective of the study was to explore and describe the experiences of mothers and caregivers on feeding practices of children under the age of five years.

\section{Methods}

This study adopted a constructivist paradigm which assisted the researcher to explore what mothers and caregivers reality with regard to feeding practices as perceived by them. ${ }^{[28]}$

\subsection{Research design}

A qualitative design was used in this study, as the researcher needed to have an in-depth understanding of the experiences of mothers and caregivers on feeding practices of children under the age of five years who developed undernutrition. ${ }^{\text {[29] }}$

\subsection{Data collection}

\subsubsection{Target population}

The population for this study were mothers and caregivers of children under the age of 5 years with moderate or severe acute malnutrition (MAM) or (SAM), either registered with Nutritional Assessment Counseling Support programme (NACS) or admitted in pediatric wards in Onandjokwe Intermediate hospital, Omuthiya and Tsumeb district hospitals in Oshikoto Region, Namibia.

\subsubsection{Sample and sampling}

A purposive sampling was used, because the researcher needed the information from people who have experienced the situation. ${ }^{[30]}$ The following inclusion criteria were considered: Mother or caregiver should for child between 0-59 months; the child should be diagnosed with MAM or SAM, registered with NACS or admitted in pediatric ward; mother or caregiver should be 18 years or older, able to make decisions (non-autonomous and mentally impaired people were excluded) and mother or caregiver should be able to speak and understand Oshiwambo (common language for Oshikoto region) or English.

The study was approved by the University of Namibia research ethics committee as well as by the Ministry of Health and Social Services. The researcher visited NACS programme and Pediatric units in Onandjokwe intermediate hospital, Omuthiya and Tsumeb district hospitals in Oshikoto region and identified mothers and caregivers who met the inclusion criteria with the assistance of programme or unit supervisors. The interview was then scheduled according to the mother or caregiver's convenient time. Participants gave a written informed consent before being interviewed. A total number of 15 mothers/caregivers were interviewed and data saturation was reached.

\subsubsection{Data collection method}

An unstructured in-depth individual interview was utilised to collect the data. This method was used, because it assisted the researcher to gain more insight and understanding of the experiences of mothers and caregivers on feeding practices of their children who developed undernutrition. ${ }^{[28]}$

Unstructured in-depth interview also helped the mothers and caregivers to talk freely about their experiences related to feeding practices. The interviews were conducted either in Oshiwambo or English, depending on the language prefer- 
ence of the participant and the data which was collected in Oshiwambo were translated into English by the researcher.

Interviews were audio-recorded with the permission of the participants, obtained through written consent to ensure accurate transcriptions. Field notes were also taken to capture important observations and non-verbal communication which cannot be audio-recorded.

\subsubsection{Measuring instrument}

An interview guide was used to collect data from the participants. The main question which was posed to each mother or caregiver who participated in this study was "What are your experiences regarding feeding practice of your child who is diagnosed with undernutrition?" Probing questions were then asked depending on the participants' responses.

\subsection{Data analysis}

Tesch's steps of open coding were adopted during data analysis and this include the following steps: ${ }^{[29]}$

- Read all the transcripts to get sense of their content and write down emerging ideas.

- Organise the emerging ideas into major and distinctive topics.

- Allocate each topic a code and write it next to the appropriate segment of text.

- Group the topics and turn them into themes.

- Categorise the data which belongs to one group in one place and refine where necessary.

\section{Results}

\subsection{Shortage of information related to nutrition and feeding practices}

Participants in this study have experienced lack of adequate information related to nutrition and feeding practices, as they do not know the types of food suitable for children under the age of five years. They attributed their inadequate information to the fact that they were not given any health education by the health workers related to nutrition and feeding practices:

"I was not told the type of food I have to give to the child; at least nurses should tell us the type of food we need to give to our children." (P\#2)

"I will strongly advise the health workers to give us some information, like you should try giving this and this, because this will also help us, instead of health workers scolding us, give us some information." (P\#8)

"We were not told anything, because once you deliver today, tomorrow you are already discharged, nobody told us about feeding of the baby, no information was given to us." (P\#14)

Published by Sciedu Press

\subsection{Usage of suboptimal feeding practices}

Mothers and caregivers who took part in the study used suboptimal feeding practices to feed their children which include early stopping of breastfeeding, bottle feeding, early introduction of complementary feeding, non-responsive feeding, less feeding frequency and poor dietary diversity. These inappropriate feeding practices contributed to children to develop undernutrition Participants in this study cease to breastfed their children early. Some of the reasons given by participants related to early breastfeeding cessation include work. Since some mothers were supposed to go to work, they stopped breastfeeding and dropped their children to grandparents. However, there were some mothers discontinued breastfeeding due their HIV status. This was communicated as follow:

"I breastfed my child till nine months, then I stopped and I took my child to my mother since I have to start working in a different town." (P\#3)

"I have been breastfeeding my baby till he turned 6 months, then from 6 months I stopped breastfeeding, then I started giving him 'mahangu' soft porridge and other milk called 'Oshitaka',” (P\#4)

"She said she was told by nurses to breastfeed till six months, so she breastfed the child for six months then she stopped." (mother is HIV positive) (P\#15)

Mothers and caregivers who took part in this study use bottles to feed their children. Some mothers and caregivers felt that it is easy to feed the child with the bottle especially during the night rather than to use spoon or cup. This was evident as follow:

"I have been using bottle to feed my baby all along, until the child was admitted to the hospital, then it was stopped when he was admitted in the hospital." (P\#12)

"I use the bottle to feed my child, I use to prepare soft porridge and put it in the bottle and add 'omayele' milk and feed her. I also use to give her 'oshikundu' using bottle, because it is easy to use the bottle especially during the night." (P\#4)

Some participants in this study started giving complementary food or water to the children as from 2-3 months. Mothers discontinued exclusive breastfeeding, because they felt that breast milk alone is not sufficient for the babies and they are not growing well. While some mothers were pressurized by their family members to start giving complementary feeding early.

"I started feeding her formula milk when she was 3 months I added formula milk, because I was not having enough breast milk, I was only breastfeeding on one breast.” (P\#3) 
"He started drinking water since birth, so I started giving him water just after few days after operation, because I gave birth through operation, so I gave him water and formula milk then he continues breastfeeding." (P\#8)

"I started adding little food when he was just a little baby, about 2 months because I use to give him some soup, since my grandmother said that we have to give him like porridge just to give him a little." (P\#12)

Participants in this study forced their children to eat in case of food refusal, so they did not feed their children responsively. Sometimes community members including nurses pressurize mothers and caregivers to force their children to eat. They explained it as follow:

"I was trying to force him to eat, I have to try and force him to open his mouth and pour in some milk, since I was forcing him, that is what causes him to have sores on the lips." (P\#5)

"Even if we tried to force him to eat, he does not want, he just wants to vomit, even when the nurses say I must force him, just force the child to eat." (P\#9)

Mothers and caregivers who took part in this study feed their children less frequently. Although participants do not possess adequate knowledge on how many times the child need to eat, sometimes the children's poor appetite has also contributed to less feeding frequencies. This was evident as follow:

"In a day, in a day I feed when we are having our lunch and dinner, that is two times per day." (P\#5)

"Sometimes I feed him three times, I give him three bottle of soft porridge, but after losing appetite, he can only take two bottles per day." (P\#4)

Mothers and caregivers who took part in this study feed their children with food from one group mainly grain. This was evident as follow:

"I started giving him 'mahangu' (pearl millet product) soft porridge till he turns 7 months and I also feed him with porridge made with mahangu flour and give him 'Oshikundu' (traditional fermented drink made from mahangu) to drink." (P\#1)

"then I use to feed her with 'mahangu' soft porridge, we stopped the soft porridge and then we started giving her 'oshikundu' made from mahangu only.” (P\#2)

\section{Discussion}

It is evident from the study that lack information related to nutrition and feeding practices as well as some other factors such as employment and family pressure have contributed to the use of inappropriate feeding practices. As a result, mothers do not breastfed their children till two years or beyond and they started complementary feeding early as reported by other researchers. ${ }^{[2,7]}$ The study revealed that mothers stop to breastfeed their children at six to nine months. One of the major concerns identified in this study is that, there are still some mothers and caregivers who are using bottle to feed their children, though bottle feeding has been discouraged by infant and young child feeding (IYF) guidelines of Namibia. ${ }^{[32]}$ Many mothers and caregivers are not able to clean bottles well and this can contribute to diarrheal diseases as reported by some authors. ${ }^{[33]}$

It was also evident that mothers and caregivers who participated in the study give complementary food to their children early at the age of two to three months. Children were given water, milk and soft porridge before they turned six months which is the recommended age for complementary feeding.

Early introduction of complementary feeding has an adverse effect on the child's health since the gut is not well developed. This study also revealed that some mothers and caregivers use non-responsive feeding, they forced their children to eat and tried hard to open their mouth and in the process some children developed sores on the lips as also stated in the literature. ${ }^{[31]}$ Some mothers and caregivers feed their children less than 2 years according to family meal schedule, though such children have already stopped breastfeeding. Children were given two to three feeds per day which resulted in unmet nutritional demands and the development of undernutrition.

Though some participants in this study indicated that they produce different types of food which include "mahangu", beans, wild spinach, pumpkins, maize, groundnuts and they also have access to some fruits such wild berries, palm fruits, fig fruits marula fruits, it has again became evident from the study the majority feed their children with food from one group, mainly grain and they consider other food not to be suitable for children, as it was also reported by other researchers. ${ }^{[26]}$ Such poor dietary diversity contributed to the development of nutritional deficiency, since children were not getting adequate nutrients. It is therefore important to provide mothers and caregivers with nutrition and feeding practices information to prevent inappropriate feeding of children under the age of five years.

\section{Conclusions}

Nutritional information plays an important role in prevention of undernutrition especially in children under the age of five years who are still in the process of growing and developing. Lack of information related to nutrition and influences from family and community members can impact on the child's 
nutritional status. This may result in irreversible outcome of undernutrition. The findings in this study showed that shortage of nutritional and feeding practices information, pressure from the relatives and employment status caused mothers and caregivers to cease breastfeeding early at the age of 6-9 months, use bottle feeding and start complementary feeding before six months.

Mothers and caregivers also feed their children less in frequency using food mainly from one group which deprived children other necessary nutrients; hence, their children developed nutrition deficiency.

It is important for the health workers to provide health information to mothers and caregivers on feeding practices of children under of five years. Such information can assist them to understand the importance of exclusive breastfeeding and the duration of breastfeeding. It can also create awareness on the dangers of bottle feeding, less frequency of feeding a child and the importance of dietary diversity. If mothers and caregivers gain knowledge related to nutrition and feeding practices, this will result in the reduction of stunted, wasted and underweight children.

This study has identified the challenges experienced by mothers and caregivers of children under the age of five years which contributed to undernutrition in their children. A contribution emanating from this study is the need to develop educational programme to empower mothers and caregivers with knowledge related to nutrition and feeding practices.

\section{Recommendations}

- Health workers need to provide mothers and caregivers with information related to nutrition and feeding practices of children from birth to five years.

- Fact sheets and posters with information related to optimal feeding practices of children under the age of five years need to be developed, distributed to mothers and displayed at antenatal departments, doctors' consulting rooms and maternity units.

\section{ACKNOWLEDGEMENTS}

We would like to acknowledge mothers and caregivers of children under the age of five years who participated in this study. We also would like to thank the Ministry of Health and Social Services for grant us a permission to conduct this study. The authors of this article contributed as follow: Ester Mulenga is the principal investigator and author of the manuscript, Hans Amukugo supervised the project and Anna Shilunga co-authored the manuscript.

\section{Conflicts of InTEREST Disclosure}

We declare that we have no financial or personal relationship which may have influenced the writing of this article.

\section{REFERENCES}

[1] Ministry of Health and Social Services. Namibia demographic and health survey 2013. Windhoek: Namibia Statistic Agency; 2014.

[2] Kulwa KBM, Verstraeten R, Bouckaert M, et al. Effectiveness of a nutrition education package in improving feeding practices, dietary adequacy and growth of infants and young children in rural Tanzania: Rationale, design and methods of cluster randomized trial. BMC Public Health [Internet]. 2014; 14: 1077. https: //doi.org/10.1186/1471-2458-14-1077

[3] Yue A, Marsh L, Zhou H, et al. Nutritional deficiencies, the absences of information and caregiver short comings: A qualitative analysis of infant feeding practice in rural China. PLoS One. 2016; 11(4). https://doi.org/10.1371/journal . pone. 0153385

[4] Sunguya BF, Poudel K, Mlunde LB, et al. Effectiveness of nutrition training of health workers toward improving caregivers' feeding practices for children aged six months to two years: A systematic review. Nutrition Journal. 2013; 12: 66. https ://doi .org/10.1186/14 75-2891-12-66

[5] Nyavani SM, Gertrude M, Flumudzani ML. Caregivers' interpretation of growth chart and feeding practices of children under five years: A case of greater Tzaneen Municipality. South Africa. Journal of Food and Nutrition Research [Internet]. 2016; 4(6): 369-376. Available from: http://pubs.sciepub.com/jfnr/4/6/5

[6] Nguyen PH, Menon P, Ruel M, et al. A situational review of infant and young child feeding practices and interventions in Vietnam. Asia
Pac Journal Clin Nutr. 2011; 20(3): 359-374. PMid:21859654

[7] Nankumbi J, Muliira JK. Barriers to infant and child feeding practices: A qualitative study of primary caregivers in rural Uganda. Journal of Health Popul Nutr. 2015; 33(1): 106-116. PMid:25995727.

[8] Kuzma J. Knowledge, attitudes and practices related to infant feeding among women in rural Pupua New Guinea: A descriptive, mixed method study. International Breastfeeding Journal [Internet]. 2013; 8: 16. PMid:24257483. Available from: http://www.internationa lbreastf eedingjournal. com/content/8/1/16

[9] Zhang K, Tang L, Wang H, et al. Why do mothers of young infants choose to formula feed in China? Perception of mothers and hospital staff. International Journal of Environment research and Public Health. 2015; 12: 4520-4532. PMid:25918908. https: //doi.org/10.3390/ijerph120504520

[10] Perera PJ, Fernando M, Warnakulasuria T, et al. Feeding practices among children attending child welfare clinics in Ragama $\mathrm{MOH}$ area: A descriptive cross-sectional study. International Breastfeeding Journal. 2011; 6: 18. https://doi .org/10.1186/1746-4358-6-18

[11] World Health Organization. Infant and young children feeding. Fact sheet. N 342. WHO; 2014.

[12] Mbagaya G. Child feeding practice in rural Western Kenya community. African Journal of Primary Health Care and Family Medicine. 2009; 1. https://doi.org/10.4102/phcfm.v1i1.15

[13] Dashti M, Scott J, Edwards CA, et al. Predictors of breastfeeding duration among women in Kuwait: Results of a prospective co- 
hort study. Nutrients [Internet]. 2014; 6: 711-728. PMid:24561360. https://doi.org/10.3390/nu6020711

[14] Semahegn A, Tesfaye G, Bogale A. Complementary feeding practices of mothers and associated factors in Hiwot Fana specialized hospital, Eastern Ethiopia. Pan African Medical Journal. 2014; 18: 143. https://doi.org/10.11604/pamj .2014.18.143.3496

[15] Matsuyama A, Karama M, Tanaka J, et al. Perceptions of caregivers about health and nutritional problems and feeding practices of infants: A qualitative study on exclusive breast-feeding in Kwale, Kenya. BMC Public Health [Internet]. 2013; 13: 525. https://doi.org/10.1186/1471-2458-13-525

[16] Shumey A, Demissie M, Berhane Y. Timely initiation of complementary feeding and associated factors among children aged 6 to 12 months in Northern Ethiopia: An institution-based crosssectional study. BMC Public Health [Internet]. 2013; 13: 105. https://doi.org/10.1186/1471-2458-13-1050

[17] Bahartha AS, AlEzzi JI. Risk factors of diarrhea in children under 5 years in Al-Mukalla. Yemen Saudi Med Journal. 2015; 36(6).

[18] Oakley LL, Henderson J, Redshaw M, et al. The role of support and other factors in early breastfeeding cessation: An analysis of data from a maternity survey in England. BMC Pregnancy and Childbirth [Internet]. 2014; 14: 88. https://doi.org/10.1186/1471-239 3-14-88

[19] Yeneabat T, Belachew T, Haile M. Determinants of cessation of exclusive breastfeeding in Ankesha Guasha Woreda, Awi zone, Northwest Ethiopia: A cross-sectional study. BMC Pregnancy \& Childbirth [Internet]. 2014; 14: 262. PMid:25107331. Available from: http://www . biomedcentral . com/1471-2393/14/262

[20] Sultana S, Hoque A, Saleh F. Infant and young child feeding practices and nutritional status in a national nutrition programme area in Bangladesh: A cross-sectional study. Journal of Human Nutrition and Food Science. 2014; 2(2): 1028.

[21] Wondafrash M, Amsalu T, Woldie M. Feeding style of caregivers of children 6-23 months of age in Derashe special district, Southern Ethiopia. BMC Public Health. 2012; 12: 235. https ://doi .org/ 10.1186/1471-2458-12-235

[22] World Health Organization. The importance of infant and young child feeding and recommended practices. Switzerland: Geneva; 2009.
[23] Benjasuwantep BJ, Chaithirayanon S, Eiamudomkan M. Feeding problem in healthy young children: Prevalence related factors and feeding practices. Pediatric Report. 2013; 5(2): 38-42. PMid:23904965. https://doi.org/10.4081/pr.2013.e10

[24] Amugsi DA, Mittelmark M, Oduro A. Association between maternal and child dietary diversity: Analysis of Ghana demographic and health survey. PLoS One. 2015; 10(8): e0136748. PMid:26305458. https://doi.org/10.1371/journal.pone. 0136748

[25] Khanal V, Sauer K, Zhao Y. Determinants of complementary feeding practices among Nepalese children aged 6-23 months: Findings from demographic and healthy survey 2011. BMC Pediatric [Internet]. 2013; 13: 131. https://doi.org/10.1186/1471-2431-13-1 31

[26] Tessema M, Belachew T, Ersino G. Feeding patterns and stunting during early childhood in rural communities of Sidama, South Ethiopia. Pan African Medical Journal. 2013; 14: 75. PMid:23646211.

[27] Lohia N, Udipi S. Infant and child feeding index reflects feeding practices status of urban slum children. BMC Pediatric [Internet]. 2014; 14: 290. https://doi .org/10.1186/s12887-014-0290-7

[28] Polit DF, Beck CT. Nursing research: Generating and assessing evidence for nursing practice. 9th ed. London, England: Lippincott Williams \& Wilkins; 2012.

[29] Creswell JW. Research Design: Qualitative, quantitative and mixed method approaches 4th ed. London: Sage Publication; 2014.

[30] Brink H, Van der Walt C, Van Rensburg G. Fundamentals of research methodology and healthcare professional. 3rd ed. Town, South Africa Juta \& Company LTD; 2012.

[31] King FS, Quinn VJ, Osei AK. Nutrition for developing countries. 3rd ed. United Kingdom: Oxford University Press; 2015. https: //doi.org/10.1093/med/9780199685226.001.0001

[32] Ministry of Health and Social Services. National policy on infant and young child feeding. Windhoek, Namibia; 2011.

[33] Das N, Chattopadhyay D, Chakraborty S, et al. Infant and young child feeding perceptions and practices among mothers in rural area of West Bengal, India. Annals of Medical \& Health Science Research. 2013; 3(3): 370-375. PMid:24116316. https ://doi .org/10.410 3/2141-9248.117955 